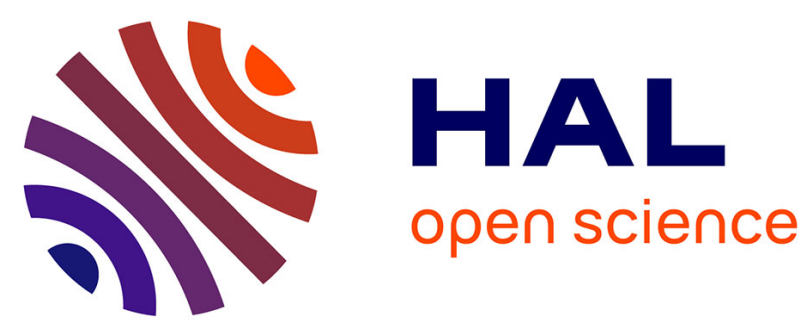

\title{
Anthropomorphic Gait Generation using Differential Dynamic Programming with a Reduced Number of Cost Criteria
}

Melya Boukheddimi, Rohan Budhiraja, Philippe Souères, Bruno Watier

\section{- To cite this version:}

Melya Boukheddimi, Rohan Budhiraja, Philippe Souères, Bruno Watier. Anthropomorphic Gait Generation using Differential Dynamic Programming with a Reduced Number of Cost Criteria. The 8th IEEE RAS/EMBS International Conference on Biomedical Robotics \& Biomechatronics, Nov 2020, New York, United States. 10.1109/BioRob49111.2020.9224427 . hal-02615813

\section{HAL Id: hal-02615813 \\ https://hal.laas.fr/hal-02615813}

Submitted on 23 May 2020

HAL is a multi-disciplinary open access archive for the deposit and dissemination of scientific research documents, whether they are published or not. The documents may come from teaching and research institutions in France or abroad, or from public or private research centers.
L'archive ouverte pluridisciplinaire HAL, est destinée au dépôt et à la diffusion de documents scientifiques de niveau recherche, publiés ou non, émanant des établissements d'enseignement et de recherche français ou étrangers, des laboratoires publics ou privés. 


\title{
Anthropomorphic Gait Generation using Differential Dynamic Programming with a Reduced Number of Cost Criteria
}

\author{
Melya Boukheddimi $^{1 *}$, Rohan Budhiraja ${ }^{2}$, Philippe Souères ${ }^{2}$ and Bruno Watier ${ }^{1}$
}

\begin{abstract}
Bipedal gait is the natural means of human locomotion. Nonetheless, it is still unclear how the central nervous system coordinates the whole-body segments for gait generation. We address this question based on the wellknown hypothesis that the human motion is the result of an optimization process. We consider a reduced set of criteria taken from the observation of human walking and the study of the related literature, which seem to be optimized during the human gait. Differential Dynamic Programming is applied on these criteria with a 3D whole-body skeletal model involving 42 degrees of freedom to generate walking motions. Nine different skeletal models and gaits reconstructed from motion capture data are used to this end. The simulated walking motions are then analyzed and compared to the human reference to show the quality of the gait generation process. The interest of this optimization approach for human-like motion generation is finally discussed.
\end{abstract}

\section{INTRODUCTION}

\section{A. Research significance}

Walking is an inherent part of human locomotion. Yet, infants acquire this ability after approximately 18 months of trial and error, going through walking on all fours. This learning process leads to a unique walking pattern characterizing each individual like a fingerprint. However, in contrast to fingerprints, learned walking patterns are not fixed once and for all, but may change according to externals variables such as accidents, pathologies or age. The centralnervous system adjusts the gait whatever the alterations [1], to allow walking in the best possible way [2]. With more than 200 Degrees of Freedom (DoFs) the human body is obviously a highly redundant system for the walking task. Hence, the issue that arises is: "what is foremost optimized by the central-nervous system in order to coordinate the entire body and generate a human gait".

\section{B. Related works}

To answer this question, active research has been conducted and different approaches were implemented. Most of them consider simplified models of poly-articulated bodies for modeling the system with rigid segments and joints. In addition some studies use a musculoskeletal model to generate gait. Inverse Kinematics was applied in [3] using motion capture (MoCap) data and muscular activations as

This work was partially supported by the European project RoboCom++, and by the French National Research agency (ANR), through the projects ANR-HOBIS (18CE27- 0010) and ANR-COBOT (18CE10 - 0003).

${ }^{1}$ LAAS-CNRS, Université de Toulouse, CNRS, UPS, France 7 Avenue du Colonel Roche, F-31400 Toulouse, France.

${ }^{2}$ LAAS-CNRS, 7 Avenue du Colonel Roche, F-31400 Toulouse, France.

*corresponding author: mboukhed@laas.fr

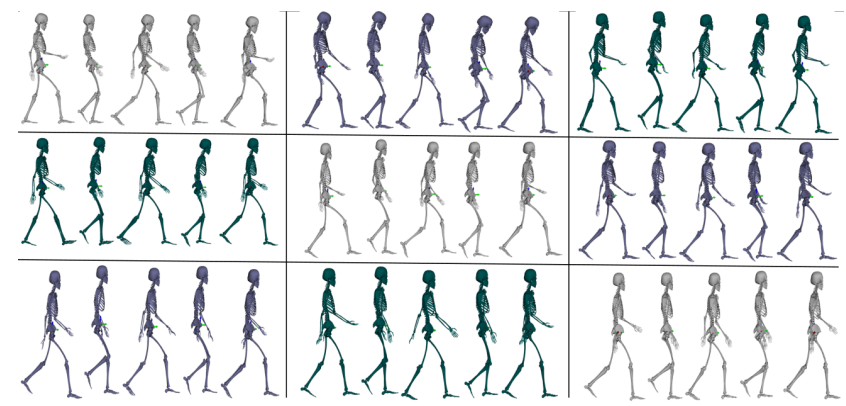

Fig. 1. Examples of human-like gaits obtained with Differential Dynamic Programming.

input. In [4] a dynamic abnormal walking was produced using muscular control with OpenSim. A neuromusculoskeletal model was used in [5] with kinematics and in vivo dynamic data to generate human-like walking and running. In [6] a neuro-physiological control of the low-level spinal was considered to simulate locomotion. With the aim of reproducing realistic human gaits, a lot of contributions were also proposed in the 3D animation community. For instance a real-time complex controller was proposed in [7] to simulate different kinds of human walking. In [8] the control framework "SYMBICON" was designed to generate bipedal walking without using MoCap data. Based on this framework, [9] presented an optimization controller robust to the environment uncertainties, which provided realistic gait simulations. A predictive human-like walking pattern was generated in [10] using a muscle-driven dynamic model with an energy optimization controller. A dynamic walking was obtained in [11] using a hierarchical tasks framework robust to the model modifications. Optimal control methods were also widely used to generate anthropomorphic gaits. One approach is to apply optimal control with direct collocation [12]. For example, in [13] gaits were generated with "tiredness and effort-like" costs optimization. Whereas in [14] the kinetics and kinematics of the gait were calculated from raw inertial sensor data and dynamic gait simulations by applying one global optimization on three cost functions. Other approaches used direct multiple-shooting to simulate walking. A predictive pattern of pathological gait applied to children was proposed in [15]. In [16], a half gait cycle was generated using a multiple optimization function approach. It was later implemented using MUSCOD II [17] and validated by comparing the obtained gait with MoCap data. In [18] direct inverse optimal control was used to identify the cost functions minimized by humans during walking by identifying the weights applied to a set of chosen criteria. 


\section{Contribution of the paper}

In this work we propose to generate human-like walking gait from optimization in accordance with [19]. We use direct optimal control with multiple-shooting to simulate human-like locomotion with multiple contacts while coping with the redundancy of a 42 DoFs model. Our objective is to demonstrate that human-like walking can be closely reproduced by optimizing a small number of key costs functions. Based on the biomechanical literature and the observations of the human gait throughout experimental analysis [20], an appropriate reduced set of optimization criteria is identified. A specific optimization method, Differential Dynamic Programming (DDP), is used to simulate the gait from the minimization of the identified criteria. Nine different models and gait patterns scaled on nine different subjects are used to test our hypothesis. Thus, Nine different simulations of gait are obtained by optimizing over the same objective criteria. Results are validated by comparing the simulated gaits profile with the human references reconstructed from MoCap considering both the kinematics and the dynamics.

\section{Structure of the paper}

The paper is organized as follows. Model and data are presented in Sec II. Sec III describes the decomposition of the human gait cycle. Sec IV exposes the optimal control (OC) problem of whole-body locomotion, and introduces the OC solver CROCODDYL. The application to gait generation is detailed in Sec V. Sec VI presents the simulation results and provides a comparison with the human reference. Finally the relevance of the approach is discussed in Sec VII.

\section{HUMAN MOTION RECONSTRUCTION}

A reconstruction of human gaits was conducted for nine volunteers, in order to construct a human reference for analyzing the human likeness of the simulated gaits.

\section{A. The data}

Experimental data were extracted from [21], they were conducted in agreement with the standards of the Declaration of Helsinki (revision 2013), and formal accordance of the ethics evaluation by the ethic committee of INSERM , Paris, France (IORG0003254, FWA00005831). The volunteers were nine healthy men, (age: $24.2 \pm 2.3 \mathrm{yrs}$, height: $1.74 \pm 0.09 \mathrm{~m}$, mass: $71.0 \pm 9.0 \mathrm{~kg}$ ) performing at least 3 trials of normal walking. One randomly chosen trial was used for each participant. The experimental framework was equipped with 13 infrared cameras sampling at 200 $\mathrm{Hz}$ (Vicon, Oxford Metrics, UK). The markers set was positioned in accordance with $\mathrm{Wu}$ guidelines [22] [23] and the International Society of Biomechanics recommendations.

\section{B. The model}

The whole-body skeletal model proposed by [24] was considered for this study as it was proven to be sufficient to reproduce the main features of human walking [20] or parkour jumps [25]. It is a 3D whole-body skeletal dynamic model involving 42 DoFs and 19 segments. This model was scaled respectively to the height and weight of each subject using the open-source software OpenSim. Therefore nine different models were obtained and used to generate nine different gaits.

\section{Reconstruction of the human reference}

An Inverse Kinematics (IK) process was conducted with the model corresponding to each subject with OpenSim [26]. The IK process aims to compute the generalized coordinates (i.e., the body configuration) that fit at best the position of the model with the experimental position of the markers at each time step. The generalized coordinates were obtained by solving a least squares problem. The resolution is based on a general quadratic programming (QP) solver [4]. The generalized coordinates obtained by solving the minimization problem will be referred to as the "human reference coordinates". They will be used in Sec VI to compare the joint angles extracted from the obtained gait simulations.

\section{GAIT ModELING}

In this section, we discuss the different contact phases that make up the human gait cycle.

\section{A. Gait cycle decomposition}

The complete anthropomorphic gait cycle is defined by two steps: left foot swinging (with right foot support) and right foot swinging (with left foot support). Each step was divided into 4 phases, according to the contact changes. Here the phases are defined only for the right foot support step; the left foot support step can be deduced by symmetry. Based on the literature of human gait dynamics, these phases are defined as shown in Fig. 2. The phases for the left foot are:

- Swinging heel of the left foot: begins when the heel of the left foot starts moving upward, and ends when the toes of the left foot take off, the left foot being no more in contact with the ground.

- Flexing of the left foot: begins when the left foot stops being in contact with the ground, and ends when the heel of the right foot loses its contact.

- Landing heel of the left foot: begins at the end of the previous phase and ends when the heel of the left foot lands on the ground.

- Landing toes of the left foot: begins when the heel of the left foot strikes the ground, the toes of the left foot moving downward, and ends when the left foot is fully in contact (6D) with the ground.

Afterwards, the next step starts with the swinging of the right foot.

\section{B. Features of the human gait}

Based on the biomechanical definition of the human gait and the related literature, the following features arise:

- The foot contact placements have to be specified to ensure the dynamic feasibility of the human gait cycle.

- During the contact changes, the three dimensional position of the center of mass (COM) of the whole-body is crucial. Notably to maintain the position of the upper part of the body at a reasonable height [21]. 


\begin{tabular}{|c|c|c|c|c|}
\hline \multicolumn{5}{|c|}{ Half cycle of gait « Right foot support » } \\
\hline $\begin{array}{c}\text { Phases of } \\
\text { motion }\end{array}$ & $\underset{\text { LF }}{\text { Swinging heel }}$ & $\begin{array}{l}\text { Flexing foot } \\
\text { LF }\end{array}$ & Landing heel LF & Landing toes LF \\
\hline Contacts & $\begin{array}{l}\text { Heel RF (6D) + } \\
\text { Toes LF (3D) }\end{array}$ & Heel RF (6D) & Toes RF (6D) & $\begin{array}{c}\text { Toes RF (3D) + } \\
\text { Heel LF (3D) }\end{array}$ \\
\hline $\begin{array}{l}\text { Beginning } \\
\text { of the } \\
\text { phase }\end{array}$ & $\begin{array}{l}\text { Foot RF on the } \\
\text { ground (Heel in } \\
\text { contact } 6 \mathrm{D} \text { ) }\end{array}$ & $\begin{array}{c}\text { Toes } L F \text { take } \\
\text { off }\end{array}$ & Heel RF takes off & Heel LF lands \\
\hline $\begin{array}{l}\text { Swinging } \\
\text { body }\end{array}$ & Heel LF & Heel LF & Heel LF & Toes LF \\
\hline
\end{tabular}

Fig. 2. Decomposition of the gait cycle. RF: right foot, LF: left foot. 3D: Three-dimensional contact. 6D: contact in translation and rotation. Red line: $3 \mathrm{D}$, purple line: $6 \mathrm{D}$.

- Flexions of the swinging knee occur when the walker tries to avoid the ground or an obstacle close to the ground with the swinging foot [27].

- Minimizing the torque is necessary to maintain a human-like torque at the joints [28].

- The velocity of the swinging foot is reduced just before landing to reduce the impact with the ground [29].

These features of the human gait and the stated cycle decomposition will be used in Sec V for simulating the gait.

\section{HUMAN GAIT EXPRESSED AS AN OPTIMIZATION PROBLEM}

\section{A. Multi-body Dynamics}

The dynamics of a poly-articulated system of rigid bodies involving $\mathrm{K}$ contacts is given by the Euler-Lagrange equations of movement (1):

$$
\mathbf{M}(q) \ddot{q}+\mathbf{b}(q, \dot{q})=\mathrm{S}^{\top} \tau+\sum_{k=1}^{K} \mathbf{J}_{k}(q)^{\top} \lambda_{k}
$$

where,

- $q$ is the configuration vector which includes the joint angles and the free-flyer coordinates.

- $\mathbf{M}(q)$ is the inertia matrix.

- $\mathbf{b}(q, \dot{q})$ contains the nonlinear and gravity forces.

- $\mathrm{S}$ is a matrix which selects the actuated components.

- $\tau$ are the internal joint torques.

- $K$ is the number of contacts.

- $\mathbf{J}_{k}(q)$ is the Jacobian matrix for the $k^{\text {th }}$ contact.

- $\lambda_{k}$ is the vector of contact forces of the $k^{t h}$ contact.

See [30] for more details.

\section{B. Rigid Contact Constraints}

During walking the body is always in contact with the ground with one or both feet. Contacts can be expressed as a kinematic constraint on the equation of movement (1). For the $k^{\text {th }}$ body the rigid contact constraint is expressed as follows:

$$
\mathbf{J}_{k} \ddot{q}+\dot{\mathbf{J}}_{k} \dot{q}=\mathbf{0} \quad \forall k \in 1 \cdots K
$$

Note that since our dynamics lies in the acceleration space the rigid contact constraint is represented as a second order kinematic constraint on the contact placement. Combining the equation of motion (1) with the contact constraints (2) the multi-contact body dynamics can be rewritten as:

$$
\left[\begin{array}{cc}
\mathbf{M} & \mathbf{J}_{c}^{\top} \\
\mathbf{J}_{c} & \mathbf{0}
\end{array}\right]\left[\begin{array}{c}
\ddot{q} \\
-\lambda_{c}
\end{array}\right]=\left[\begin{array}{c}
\mathbf{S}^{\top} \tau-\mathbf{b} \\
-\mathbf{J}_{c} \dot{q}
\end{array}\right]
$$

where $^{1}, \mathbf{J}_{c}=\left[\mathbf{J}_{0}^{\top} \cdots \mathbf{J}_{k}^{\top} \cdots \mathbf{J}_{K}^{\top}\right]^{\top}$ and $\lambda_{c}=\left[\lambda_{0}^{\top} \cdots \lambda_{k}^{\top} \cdots \lambda_{K}^{\top}\right]^{\top}$

This redefined dynamics is the same as the one obtained by applying the Gauss principle of least constraints on the motion of a multi-body system with contact constraints [32]. If we consider the Karush-Kahn-Tucker optimality conditions on the Gauss's principle, we obtain (3) [33].

\section{Optimization for motion generation: Crocoddyl Solver}

Consider the problem of generating a human-like gait using the dynamics defined in Sec IV-A and IV-B. To this end a multi-phase optimization problem is defined, each phase being governed by (3) under a specific set of contact. The fixed contacts and the gait modeling was discussed in Sec III. The optimization problem is formulated as ${ }^{2}$ :

$$
\begin{array}{cl}
\underset{q, \dot{q}, \tau}{\operatorname{minimize}} & \sum_{s=1}^{S} \int_{t_{s}}^{t_{s}+\Delta t_{s}} l_{S}(q, \dot{q}, \tau) d t \\
\text { subject to } & q \in \mathscr{Q} \\
& \dot{q} \in \mathscr{V} \\
& \tau \in \mathscr{T} \\
& {[\dot{q}, \ddot{q}]^{\top}=f(q, \dot{q}, \tau)}
\end{array}
$$

where,

- $s=1 \cdots S$ is used to describe the contact phases which define the human walking gait, as discussed in Sec III.

- $l_{s}$ is the cost function for the contact phase $s$.

- $f$ is a reduced formulation of the contact constrained dynamics (3).

- $\mathscr{Q}, \mathscr{V}, \mathscr{T}$ are the admissible sets for $q, \dot{q}, \tau$ (defined by the biological limits of the human joint).

To solve this optimization problem we use a Shooting Method. These methods provide a way of solving boundary value problems with simple path constraints (such as (4)). Shooting methods are popular since they are fast to solve compared to other available methods [12]. Among Shooting methods, Differential Dynamic Programming (DDP) is a good way of handling this optimization problem. DDP allows us to truly exploit the sparsity introduced by the Markovian nature of the dynamics in (3) in a highly iterative manner. In this work, we use the framework Crocoddyl [34] to solve the above optimization problem. Crocoddyl is a DDP-based library [33], which is open-Source, memory efficient and uses fast dynamics and derivative algorithms provided by Pinocchio [35]. Moreover, Crocoddyl proposes a variant of DDP, called FDDP (Feasibility DDP) [36], which avoids the numerical limitations of single-shooting in DDP and allows warm starting the problem from an infeasible

\footnotetext{
${ }^{1}$ When considering the rigid contact constraint (2) in the contact constrained dynamics (3), terms defined by Baumgarte Stabilization [31] are often included to improve numerical behaviour of integration.

${ }^{2} q, \dot{q}, \ddot{q}, \tau$ are functions of $t$. We drop the dependence here for clarity.
} 
state. In this research, we use the FDDP method to solve the discretized version of our optimization problem (4).

\section{Gait Simulation}

Relying on the observations made in Sec III-B and according to the decomposition of the gait cycle stated in Sec IIIA, we define an optimization problem that covers each step. Solving this optimization problem leads to compute the optimal trajectories of the state $x=(q, \dot{q})$, and the optimal control $u=\tau$. Moreover, our formulation (3) leads to compute the contact forces $\lambda_{k}$ as a function of $x$ and $u$.

In order to solve our optimization problem numerically with Crocoddyl, the problem is first discretized over $N$ subintervals (evenly distributed or not) of $[0, T]$. In each subinterval $n$, the control trajectory $u_{n}$ is constrained to be in the span of a given finite trajectory basis, and is fixed to a constant value. The values of $x_{n}$ are obtained by integrating numerically the dynamics (3) using an Implicit Euler scheme [37]. In Crocoddyl, we define a separate dynamics and cost model (collectively called an action model) for each node of the descritization interval, the optimization problem is created as a list of action models in a series. Based on the list of features of the human gait listed in Sec III-B different costs $\Phi_{n}$ are designed for the optimization problem. Each cost is weighted differently by a coefficient $\alpha_{n} \in \mathbb{R}$. The final objective function for an action model is written as:

$$
l_{s}=\sum_{n=1}^{N} \alpha_{n} \Phi_{n}(q, \dot{q}, \tau),
$$

with the following costs and weights, $t_{s}$ denoting the final time of each phase, and $t_{\text {final }}$ the final time of each half cycle of the gait.

- CoM Cost: During a contact phase, the CoM trajectory $c(t)$ tracks the final CoM position for each phase $c^{r e f}\left(t_{s}\right)$.

$\Phi_{1}=\left\|c(t)-c^{r e f}\left(t_{s}\right)\right\|_{2}^{2}, \quad \alpha_{1}=17 \times 10^{3}$

- Feet Cost: During a contact phase, the swinging foot position trajectory $\left(r_{h}(t)\right.$ for the heel or $r_{t}(t)$ for the toes) tracks the final foot placement for each phase. $\Phi_{2}=\left\|r_{h}(t)-r_{h}^{r e f}\left(t_{s}\right)\right\|_{2}^{2}, \quad \alpha_{2}=1 \times 10^{3}$

- Ground Avoidance: The swinging foot needs to maintain a clearance from the ground. This is implemented by a quadratic barrier on the $z$ position of the foot.

$\Phi_{3}=\left\|\operatorname{minimum}\left\{r_{h}^{z}(t)-r_{h}^{z}\left(t_{s}\right), 0\right\}\right\|_{2}^{2}, \quad \alpha_{3}=50 \times 10^{3}$

- Minimization of the toes velocity: $\left(\dot{r}(\text { toes })_{t_{\text {final }}}\right)$ just before the impact of the swinging foot (applicable only on the toes and on the last node of a half cycle of gait) $\Phi_{4}=\| \dot{r}(\text { toes })_{t_{\text {final }}} \|_{2}^{2}, \quad \alpha_{4}=1 \times 10^{5}$

- Torque minimization: Minimization of the joint torques for realistic dynamic movements.

$\Phi_{5}=\|\tau(t)\|_{2}^{2}, \quad \alpha_{5}=1 \times 10^{-3}$

- Posture regularization: This cost deals with the redundancy of multi-body dynamics, giving as input only the final reference posture for each phase.

$$
\Phi_{6}=\left\|q(t)-q^{r e f}\left(t_{s}\right)\right\|_{2}^{2}, \quad \alpha_{6}=0.1
$$

Just for the final node of a half cycle of gait at $t_{\text {final }}$, a higher weight $\alpha=10$ is used for the posture task. The

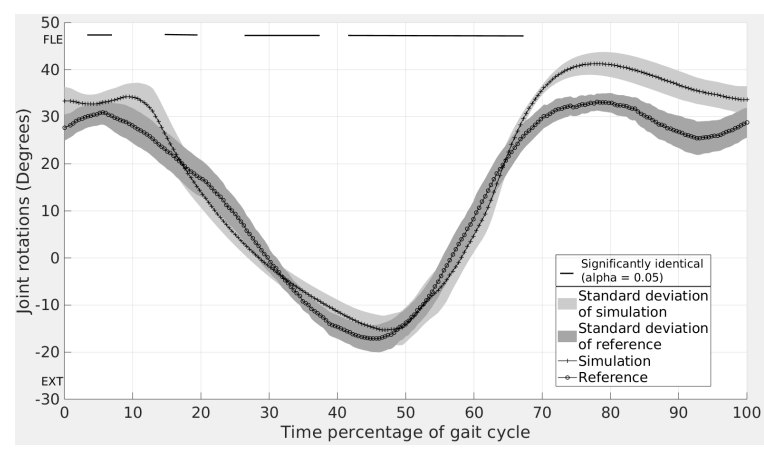

Fig. 3. Flexion-extension of the right hip

weights $\alpha_{i}$ for this optimization problem were determined experimentally. Their were adjusted in order to obtain the better kinematic results, which were assessed by comparing them to the joint rotations obtained from MoCap. Additionally to this kinematic benchmarking, the joint torques were also checked and the costs $\Phi_{5}$ were tuned with the aim to obtain human-like joints torques values. The obtained results are validated in the next section by comparing them to the human references coming from MoCap, using the following biomechanical criteria:

- Range Of Motion (ROM) of the joint rotations.

- Mean and standard deviation.

- Student test $(\alpha=0.05)$, with his test of significance.

- Shapiro-Wilk test of normality ( $\alpha=0.05$ ).

\section{RESULTS AND COMPARISON}

\section{A. Results}

Nine anthropomorphic gait were generated, each one with its own scaled model and gait pattern. These simulations were obtained by solving the same optimization problem defined in Sec V and III for each gait cycle. Some sequences of snapshots of complete gait-cycles are presented in Fig. 1. Relevant human-like gait behaviors arise from these simulations. Indeed, the generated movements show a human-like roll of the swinging foot on the ground, and a natural flexion of both the knees and the hip. Regarding the upper part of the body including the neck and the head, a good tracking of the human walking pattern with natural back and forth motions of the arms can be observed. Additionally to these visual and qualitative observations, a statistical analysis is provided in the next section.

\section{B. Comparison}

This section summarizes the study that was conducted to compare the simulated gaits obtained from the resolution of the OC problem and the reference human movement. We compute the mean and standard deviation of joint rotations over one gait cycle for the nine subjects. Fig. 3 to Fig. 8 show the simulated and the reference gaits for the right lower limbs, i.e the hip, the knee and the ankle. For all the obtained movements the results indicate that big parts of the motion are significantly identical to the reference movements. Table I presents, for the simulated gait and the human reference, the mean of the ROM for the joint rotations 


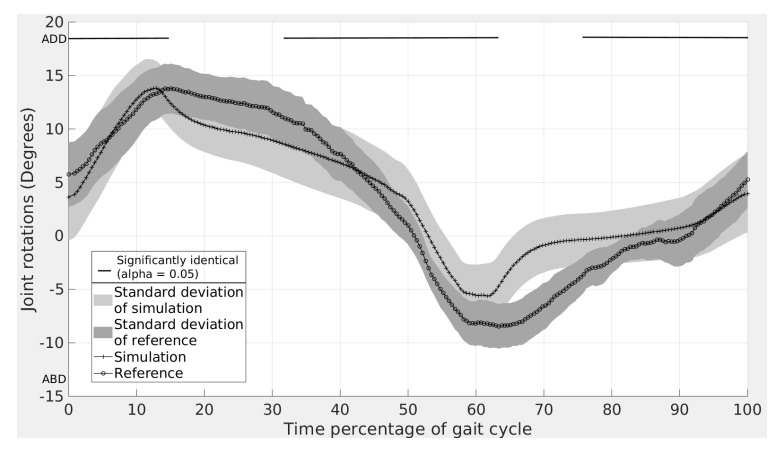

Fig. 4. Abduction - adduction of the right hip

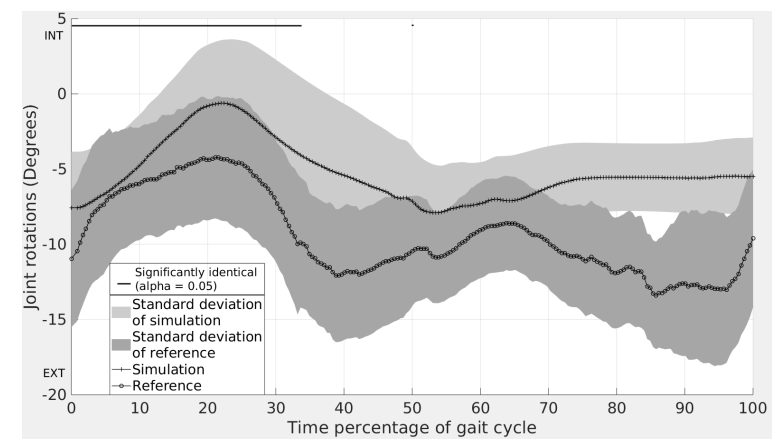

Fig. 5. External-internal rotation of the right hip

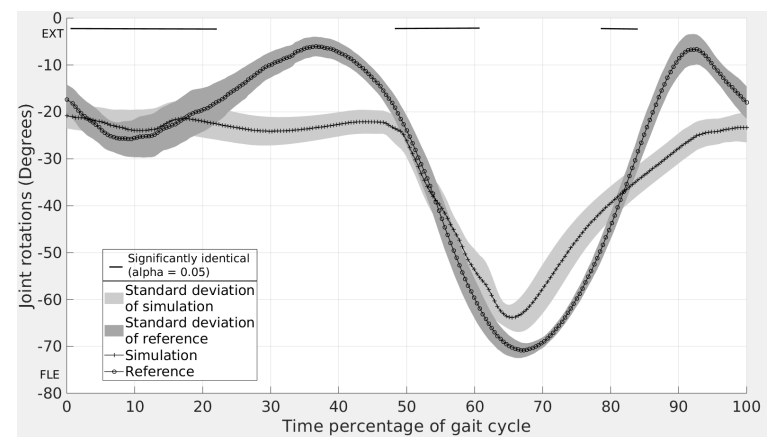

Fig. 6. Flexion-extension of the right knee

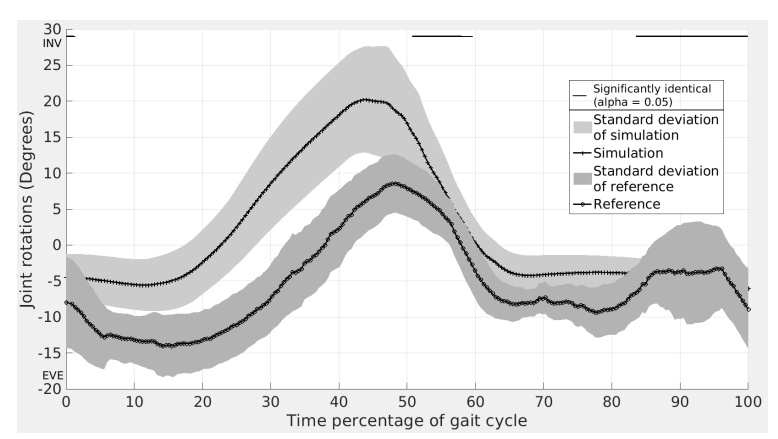

Fig. 7. Eversion-inversion of the right ankle

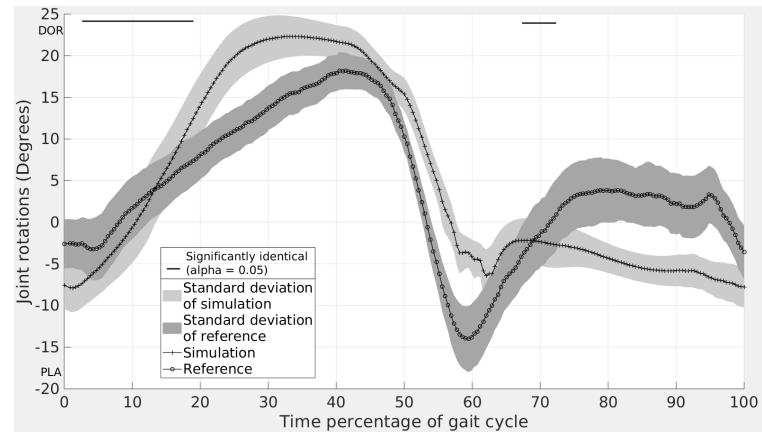

Fig. 8. Plantarflexion-dorsiflexion of the right ankle

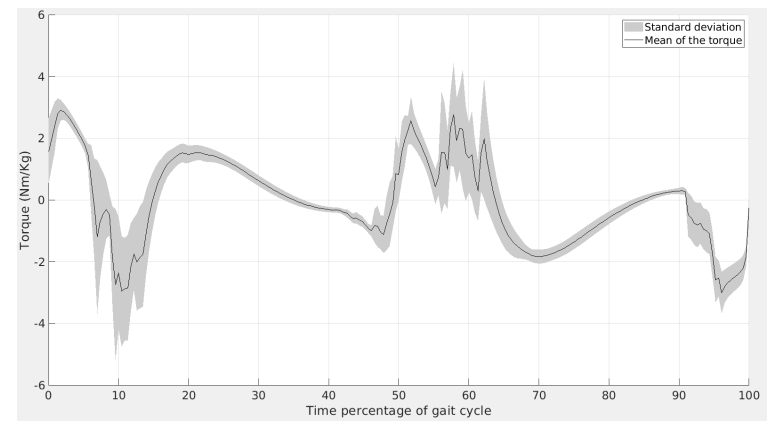

Fig. 9. Mean and standard deviation of the joint torques of the flexionextension of the right hip.

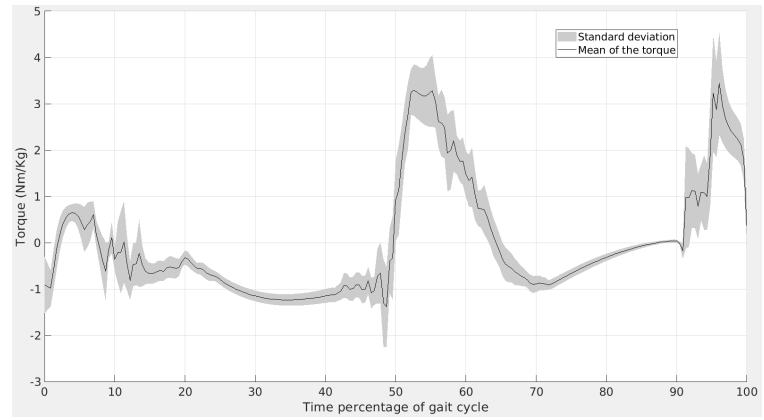

Fig. 10. Mean and standard deviation of the joint torques of the flexionextension of the right knee.

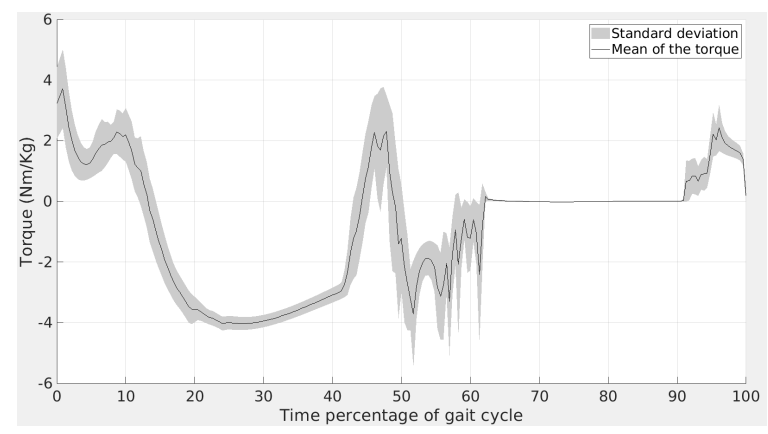

Fig. 11. Mean and standard deviation of the joint torques of the plantarflexion-dorsiflexion of the right ankle. 


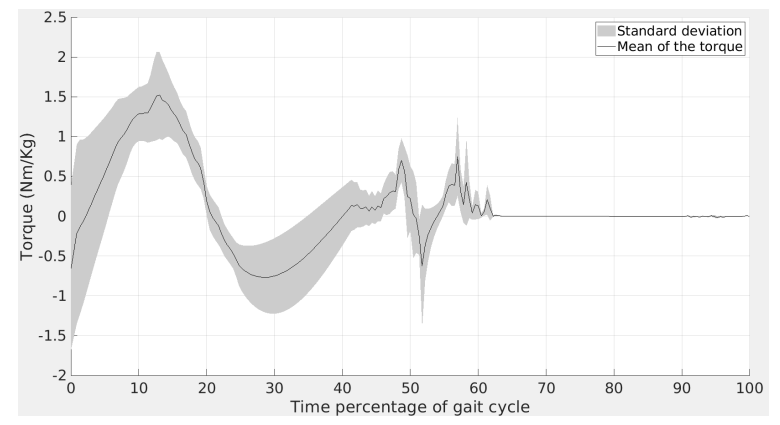

Fig. 12. Mean and standard deviation of the joint torques of the eversioninversion of the right ankle.

TABLE I

THE MEANS OF THE ROM FOR THE JOINT ROTATIONS (DEGREES). \$ SIGNIFICANTLY IDENTICAL $(\alpha=0.05)$. R : RIGHT, L : LEFT.

\begin{tabular}{|l|c|c|c|}
\hline \multicolumn{1}{|c|}{ Joint Rotations } & $\begin{array}{c}\text { ROM } \\
\text { Simulation }\end{array}$ & $\begin{array}{c}\text { ROM } \\
\text { Reference }\end{array}$ & $\begin{array}{c}\text { ROM } \\
\text { Difference }\end{array}$ \\
\hline HIP R Flex/Exte & 59 & 54 & 5 \\
\hline HIP R Abdu/Aduc & 23 & 24 & $1 \neq$ \\
\hline HIP R Axial rotation & 12 & 16 & $4 \ddagger$ \\
\hline Knee R Flex/Exte & 51 & 67 & 16 \\
\hline Ankle R Dors/Plan & 36 & 34 & $2 \ddagger$ \\
\hline Ankle R Ever/Inve & 30 & 27 & $3 \ddagger$ \\
\hline Hip L Flex/Exte & 62 & 57 & $5 \ddagger$ \\
\hline Hip L Abdu/Aduc & 26 & 26 & $0 \ddagger$ \\
\hline Hip L Axial rotation & 13 & 16 & $3 \ddagger$ \\
\hline Knee L Flex/Exte & 48 & 66 & 18 \\
\hline Ankle L Dors/Plan & 35 & 35 & $0 \ddagger$ \\
\hline Ankle L Ever/Inve & 29 & 29 & $0 \ddagger$ \\
\hline
\end{tabular}

of the lower limbs with their differences. The Student test is conducted and the obtained joint rotations which are significantly identical to the references are highlighted in this table. The ROM for the simulated joint rotations are significantly identical to the human reference for the ankle, the abduction - adduction and the external-internal rotation of the hip for both left and right sides, and for the flexionextension of the left hip. Regarding this comparison, the results show that the joint rotations of the simulated gaits closely follow the joint rotations pattern and values of the human references. Moreover, for the nine subjects, the mean and standard deviation of the joint torques of the right lower limbs, obtained in simulation over one gait cycle, are represented in Fig. 9 to 12 . It can be noticed that the simulated joint torques remain within the physiological limits: mostly between -1 and $1 \mathrm{Nm} \cdot \mathrm{Kg}^{-1}$, with maximum values $\pm 4 \mathrm{Nm} \cdot \mathrm{Kg}^{-1}$. However, these joint torques are not following a human-like profile along the gait cycle, as described in [2].

\section{CONCLUDING DISCUSSION}

The analysis of the simulated walking movement presented in Sec VI supports the hypothesis stated in (Sec IC), namely that the anthropomorphic gait can be reproduced from the optimization of a small number of adequately chosen criteria. In our approach only 8 phases were used to reconstruct the full gait cycle, while only discrete values at the end of each phase were given to the optimizer, without requiring any reference trajectories. By comparison, a half gait cycle required 5 phases to be simulated in [16]. Having a low number of phases makes the method more general and easier to implement as the quantity of data required to specify the motion is reduced. Concerning the dynamics, the only considered optimization criterion is the torque minimization which provides a way of regularizing the optimization problem. As a result we observed that the torques values remain within the physiological limits of the human movement all along the gait cycle. In our previous work [20], in which the task-hierarchy framework was used with a kinematic model, it was necessary to give the highest priority to the foot tracking task. Here, as the full body dynamics is considered, a good quality of the trajectories is obtained by putting a higher weight on the CoM cost than on the foot cost. This difference reflects the key role of the CoM regulation in dynamical systems. The proposed method based on DDP allows the minimization process to be handled for at least one complete gait cycle while preserving a good quality of the optimized movement including the execution of the human-like features all along the simulation. The strength of DDP lies in its ability to solve the problem in a highly iterative and fast manner. However, in order to produce quick results, DDP compromises in its ability to consider constraints on the optimization (unlike previous works which used Non-Linear Programming solvers like MUSCOD-II [17]). While the ability to consider constraints is definitely a plus, as shown by [18], we found that we can by-pass the constraints by using penalty methods on DDP. Moreover, the additional computational speed offered by DDP opens new avenues in which to carry our research. While speed of computation is more relevant in control of systems, the fast results by DDP give us the ability to transition smoothly from the realm of biomechanics to that of robotics. Thus, this work could possibly be extended to apply our biomechanical analysis in hardware system, in order to generate realistic humanlike movements in real time. Finally, though the generated gaits show some important anthropomorphic features of the human walking, we believe that the following propositions provide interesting directions for further improving our results: (1) Adding a cost for the contact forces to the optimization problem could lead to better movements, and be suitable for dynamic validation in a physics engine. (2) A sufficient flexion of the swinging knee was obtained by the Ground Clearance criterion. Removing this cost results in a stiff-knee with an insufficient flexion. This problem could be alleviated by adding instead a cost for the CoM to follow the Spring-Load Inverted Pendulum dynamics. (3) Since we did not try to constraint directly the knee, the maximal extension of this joint, especially during stance phase, was not reached in the simulations. A dynamic cost based on the correlation between the contact forces during the impact and the extension of the knee could be considered in future works. (4) The weights in the optimization problem were 
so far determined empirically. An inverse optimal control method could be used to compute this weights (as it was formulated in [38]). This is indeed a research for the future.

\section{REFERENCES}

[1] M. L. Latash, "The bliss (not the problem) of motor abundance (not redundancy)," Experimental Brain Research, vol. 217, no. 1, pp. 1-5, Mar 2012.

[2] E. Viel, La marche humaine, la course et le saut: biomécanique, explorations, normes et dysfonctionnements, ser. Le Point en rééducation. Masson, 2000.

[3] A. Rajagopal, C. L. Dembia, M. S. DeMers, D. D. Delp, J. L. Hicks, and S. L. Delp, "Full-body musculoskeletal model for muscle-driven simulation of human gait," IEEE Trans on Biomedical Engineering, vol. 63, no. 10, pp. 2068-2079, Oct 2016.

[4] S. Delp, F. Anderson, A. Arnold, P. Loan, A. Habib, C. John, E. Guendelman, and D. Thelen, "Opensim: Open-source software to create and analyze dynamic simulations of movement," Biomedical Engineering, IEEE Trans on, vol. 54, pp. 1940 - 1950, 122007.

[5] K. Hase, K. Miyashita, S. Ok, and Y. Arakawa, "Human gait simulation with a neuromusculoskeletal model and evolutionary computation," The Journal of Visualization and Computer Animation, vol. 14, no. 2, pp. 73-92, 2003.

[6] S. Song and H. Geyer, "A neural circuitry that emphasizes spinal feedback generates diverse behaviours of human locomotion," The Journal of physiology, vol. 593, no. 16, pp. 3493-3511, 2015.

[7] S. Coros, P. Beaudoin, and M. van de Panne, "Generalized biped walking control," ACM Transctions on Graphics, vol. 29, no. 4, p. Article 130, 2010.

[8] K. Yin, K. Loken, and M. van de Panne, "Simbicon: Simple biped locomotion control," ACM Trans. Graph., vol. 26, no. 3, Jul. 2007.

[9] J. M. Wang, S. R. Hamner, S. L. Delp, and V. Koltun, "Optimizing locomotion controllers using biologically-based actuators and objectives," ACM Trans. Graph., vol. 31, no. 4, pp. 25:1-25:11, Jul. 2012.

[10] T. W. Dorn, J. M. Wang, J. L. Hicks, and S. L. Delp, "Predictive simulation generates human adaptations during loaded and inclined walking," PLOS ONE, vol. 10, no. 4, pp. 1-16, 042015.

[11] M. de Lasa, I. Mordatch, and A. Hertzmann, "Feature-Based Locomotion Controllers," ACM Trans on Graphics, vol. 29, no. 3, 2010.

[12] M. Diehl and S. Gros, "Numerical optimal control (preliminary and incomplete draft)," 2017.

[13] M. Ackermann and A. J. van den Bogert, "Optimality principles for model-based prediction of human gait," Journal of Biomechanics, vol. 43, no. 6, pp. 1055 - 1060, 2010.

[14] E. Dorschky, M. Nitschke, A.-K. Seifer, A. J. van den Bogert, and B. M. Eskofier, "Estimation of gait kinematics and kinetics from inertial sensor data using optimal control of musculoskeletal models," Journal of Biomechanics, vol. 95, p. 109278, 2019.

[15] M. Sreenivasa, M. Millard, M. Felis, K. Mombaur, and S. I. Wolf, "Optimal control based stiffness identification of an ankle-foot orthosis using a predictive walking model," Frontiers in Computational Neuroscience, vol. 11, p. 23, 2017.

[16] M. L. Felis and K. Mombaur, "Synthesis of full-body 3-d human gait using optimal control methods," in 2016 IEEE (ICRA), May 2016, pp. $1560-1566$.

[17] P. Kuhl, J. Ferreau, J. Albersmeyer, C. Kirches, L. Wirsching, S. Sager, A. Potschka, G. Schulz, M. Diehl, D. Leineweber, and A. Schafer, "Muscod-ii users' manual," 012001.

[18] D. Clever, R. Malin Schemschat, M. L. Felis, and K. Mombaur, "Inverse optimal control based identification of optimality criteria in whole-body human walking on level ground," in IEEE International Conference BioRob, June 2016, pp. 1192-1199.

[19] E. Todorov and W. Li, "Optimal control methods suitable for biomechanical systems," in Proceedings of the 25th Annual International Conference of the IEEE Engineering in Medicine and Biology Society (IEEE Cat. No. 03CH37439), vol. 2. IEEE, 2003, pp. 1758-1761.

[20] M. Boukheddimi, F. Bailly, P. Souères, and B. Watier, "Human-like gait generation from a reduced set of tasks using the hierarchical control framework from robotics," in IEEE Conference on Robotics and Biomimetics, Dali, China, Dec 2019.

[21] J. Carpentier, M. Benallegue, and J.-P. Laumond, "On the centre of mass motion in human walking," Int. J. Autom. Comput., vol. 14, no. 5, pp. 542-551, Oct. 2017.
[22] G. Wu, S. Siegler, P. Allard, C. Kirtley, A. Leardini, D. Rosenbaum, M. Whittle, D. D. DLima, L. Cristofolini, H. Witte, O. Schmid, and I. Stokes, "Isb recommendation on definitions of joint coordinate system of various joints for the reporting of human joint motionpart i: ankle, hip, and spine," Journal of Biomechanics, vol. 35, no. 4, pp. $543-548,2002$.

[23] G. Wu, F. van der Helm, H. Veeger, M. Makhsous, P. van Roy, C. Anglin, J. Nagels, A. Karduna, K. McQuade, X. Wang, F. Werner, and B. Buchholz, "Isb recommendation on definitions of joint coordinate systems of various joints for the reporting of human joint motion-part ii: shoulder, elbow, wrist and hand," Jr of Biomechanics, vol. 38, pp. 981-92, 2005.

[24] G. Maldonado, P. Souères, and B. Watier, "Whole-body musculoskeletal model v1," Université de Toulouse 3 Paul Sabatier ; LAASCNRS, Technical Report Rapport LAAS n 18233, 2018.

[25] G. Maldonado, F. Bailly, P. Souères, and B. Watier, "Angular momentum regulation strategies for highly dynamic landing in Parkour," Computer Methods in Biomechanics and Biomedical Engineering, vol. 20, no. sup1, pp. 123-124, 2017.

[26] S. R. Hamner, A. Seth, and S. L. Delp, "Muscle contributions to propulsion and support during running," Journal of Biomechanics, vol. 43, no. 14, pp. 2709 - 2716, 2010.

[27] J. M. Byrne and S. D. Prentice, "Swing phase kinetics and kinematics of knee replacement patients during obstacle avoidance," Gait \& Posture, vol. 18, no. 1, pp. 95-104, aug 2003.

[28] Y. Xiang, J. S. Arora, and K. Abdel-Malek, "Optimization-based prediction of asymmetric human gait," Journal of Biomechanics, vol. 44 , no. 4 , pp. $683-693,2011$.

[29] W. Kim, A. S. Voloshin, and S. H. Johnson, "Modeling of heel strike transients during running," Human Movement Science, vol. 13, no. 2, pp. $221-244,1994$.

[30] R. Featherstone, Rigid Body Dynamics Algorithms. Springer US, 2014. [Online]. Available: https://books.google.fr/books?id= GJRGBQAAQBAJ

[31] J. Baumgarte, "Stabilization of constraints and integrals of motion in dynamical systems," Computer Methods in Applied Mechanics and Engineering, vol. 1, no. 1, pp. 1-16, jun 1972.

[32] P.-B. Wieber, "Holonomy and nonholonomy in the dynamics of articulated motion," in Proceedings of the Ruperto Carola Symposium on Fast Motion in Biomechanics and Robotics, 2005.

[33] R. Budhiraja, J. Carpentier, C. Mastalli, and N. Mansard, "Differential Dynamic Programming for Multi-Phase Rigid Contact Dynamics," in IEEE-RAS Humanoids 2018, Beijing, China, Nov. 2018.

[34] C. Mastalli, R. Budhiraja, W. Merkt, G. Saurel, B. Hammoud, M. Naveau, J. Carpentier, L. Righetti, S. Vijayakumar, and N. Mansard, "Crocoddyl: An Efficient and Versatile Framework for Multi-Contact Optimal Control," May 2020.

[35] J. Carpentier, G. Saurel, G. Buondonno, J. Mirabel, F. Lamiraux, O. Stasse, and N. Mansard, "The Pinocchio C++ library - A fast and flexible implementation of rigid body dynamics algorithms and their analytical derivatives," in SII 2019 - International Symposium on System Integrations, Paris, France, jan 2019.

[36] N. Mansard, "Feasibility-prone differential dynamic programming is ddp a multiple shooting algorithm?)," 2019. [Online]. Available: https://gepgitlab.laas.fr/loco-3d/crocoddyl

[37] W. H. Press, S. Teukolsky, W. Vetterling, and B. Flannery, Numerical recipes : the art of scientific computing, $3 \mathrm{rd}$ ed. Cambridge University Press, sep 2007.

[38] A. M. Panchea, N. Ramdani, V. Bonnet, and P. Fraisse, "Human Arm Motion Analysis Based on the Inverse Optimization Approach," in International Conference Biorob. Enschede, Netherlands: IEEE, 2018, pp. 1005-1010. 